\title{
Measurement of the ultrafast gain recovery in InGaAs/GaAs quantum dots: Beyond a mean-field description
}

\author{
Paola Borri, ${ }^{1,2, *}$ Valentina Cesari, ${ }^{2}$ and Wolfgang Langbein ${ }^{2}$ \\ ${ }^{1}$ School of Biosciences, Cardiff University, Museum Avenue, Cardiff CF10 3AX, United Kingdom \\ ${ }^{2}$ School of Physics and Astronomy, Cardiff University, The Parade, Cardiff CF24 3AA, United Kingdom
}

(Received 29 August 2010; published 27 September 2010)

\begin{abstract}
We measured the ultrafast gain recovery dynamics of the ground-state transition in an ensemble of electrically pumped InGaAs/GaAs quantum dots having a nonequilibrium carrier distribution prepared by an optical prepump pulse. We find that the gain recovery dynamics after optical depletion by the prepump is faster than without prepump, an effect most pronounced at low temperature $(15 \mathrm{~K})$ but observable up to room temperature. This finding is not consistent with a mean-field description of the carrier distribution and gives direct evidence that microstates with discrete carrier numbers determine the macroscopic response. The observed dynamics results from a conditional recovery where microstates with an internal relaxation slower than the depletion lead time are selectively suppressed.
\end{abstract}

DOI: 10.1103/PhysRevB.82.115326

PACS number(s): 78.67.Hc, 78.47.jb, 78.47.N-

\section{INTRODUCTION}

Understanding how the localization of electrons and holes in semiconductor nanostructures is affecting their dynamics is of interest to fundamental many-body physics but is also key to applications in high-speed optoelectronics. Semiconductor quantum dots (QDs) confine carriers on the nanometer scale in all three dimensions. They have attracted growing interest over the past fifteen years, and their potential for improved-performance devices such as lasers and amplifiers has been demonstrated. ${ }^{1}$ One important question in the description of the carrier distribution in such smallvolume structures is whether macroscopic observables are well described by a mean-field theory that uses average state populations over the ensemble of configurations (originating from many QDs or a time ensemble over many repeats in a single QD). In the literature describing the dynamical behavior of carriers in QD lasers/amplifiers the mean-field approach is widely utilized with various levels of sophistication from simple rate equation models to semiconductor Bloch equations. $^{2-4}$ On the other hand, it was pointed out in Ref. 5 that when considering QD ensembles in the low-temperature limit in which thermal re-emission of a carrier from a QD is much slower than carrier recombination, the assumption of a population-averaged Fermi distribution function across the ensemble is flawed. Instead the macroscopic ensemble has to be described as a superposition of microstates, where a microstate is defined by the occupation of a QD with a given discrete number of carriers and its probability is evolving according to master equations. This model predicts a nonthermal carrier distribution where dots with different groundstate (GS) energies are equally occupied on average, due to the lack of interdot coupling at low temperature and the randomness of carrier capture and recombination events. Such prediction was indeed supported by the experimental evidence of a minimum in the temperature dependence of the threshold current in QD lasers ${ }^{6}$ and in the spectral linewidth of inhomogeneously broadened QD ensembles. ${ }^{7}$ In single quantum dots the power dependence of the photoluminescence (PL) emission of exciton complexes at low tempera- ture has also been explained using microstates. ${ }^{8,9}$ In terms of carrier dynamics, it was shown that the time-resolved PL from multiexcitons in single quantum dots behaves as a cascade evolution of radiative recombination processes since at any given time only one well defined multiexciton (i.e., microstate) may exist in the QD. ${ }^{10-12}$

However, up to now no experiment has been designed to reveal the failure of the mean-field description for the gain dynamics of QD lasers/amplifiers and a direct manifestation of the microstate nature of intradot carrier relaxation in QDs is still missing. In this work, we provide direct evidence that the macroscopic response of the gain recovery dynamics in electrically pumped QD ensembles is a superposition of intradot relaxation dynamics from microstates with discrete numbers of carriers. Using dual-pump probe differentialtransmission spectroscopy, we can selectively prepare the ensemble suppressing microstates with slow internal relaxation dynamics, and demonstrate ultrafast conditional gain recovery not explained by mean-field theory.

\section{SAMPLE AND EXPERIMENT}

The investigated sample is a $p$-type-intrinsic- $n$-type (pi-n) ridge waveguide structure of $4 \mu \mathrm{m}$ width and $0.5 \mathrm{~mm}$ length containing $10 \mathrm{InGaAs}$ dot-in-well layers separated by $33 \mathrm{~nm}$ GaAs spacers and sandwiched between $1.5 \mu \mathrm{m}$ thick AlGaAs cladding layers (see sketch in Fig. 1). The waveguide was processed with tilted facets $\left(\sim 7^{\circ}\right)$ to avoid back reflection into the waveguide mode and lasing. Amplified spontaneous emission spectra at room temperature ${ }^{13}$ showed GS excitonic emission near $1.3 \mu \mathrm{m}$ wavelength with $\sim 36 \mathrm{meV}$ inhomogeneous broadening and emission from the first optically active excited-state (ES) transition at 60 meV above the GS. The equilibrium carrier density in the QDs, and accordingly the gain coefficient $\left(g_{0}\right)$ of the amplifier at the GS transition, was controlled by the injection current applied to the $\mathrm{p}-\mathrm{i}-\mathrm{n}$ diode. ${ }^{13,14}$ The device temperature was adjusted between 15 and $300 \mathrm{~K}$ in a cold-finger cryostat with custom-designed windows to enable optical coupling. 


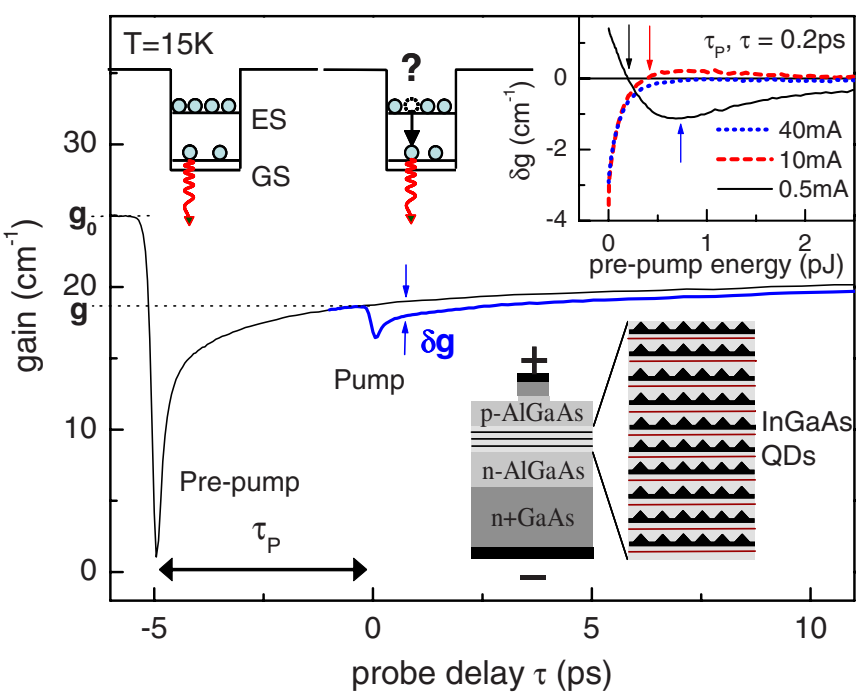

FIG. 1. (Color online) Transient GS gain change in InGaAs QDs at $40 \mathrm{~mA}$ injection current at $15 \mathrm{~K}$ as function of the delay time between pump and probe optical pulses, in the presence of a strong prepump fully depleting the GS gain. The differential change in the gain due to the second pump is indicated as $\delta g$. A sketch of the sample is shown. In the inset $\delta g$ measured for 0.2 ps prepump $\left(\tau_{\mathrm{P}}\right)$ and probe $(\tau)$ delays is shown versus prepump pulse energy for different injection currents corresponding to GS absorption (0.5 $\mathrm{mA})$ and gain $(10 \mathrm{~mA}, 40 \mathrm{~mA})$. Arrows indicate the values of prepump pulse energies chosen in the transient dual-pump probe experiments.

Gain dynamics were measured by pump-probe differential transmission in heterodyne detection similar to our previous works, ${ }^{15}$ extended here to a dual pump scheme. In this technique, $\sim 100$ fs Fourier-limited pulses at $76 \mathrm{MHz}$ repetition rate, with an optical center frequency in resonance with the GS transition energy, are divided into a prepump, a pump, a probe, and a reference beam. Prepump, pump, and probe beams are shifted by radio-frequency amounts of $81 \mathrm{MHz}$, $80 \mathrm{MHz}$, and $79 \mathrm{MHz}$, respectively, using acousto-optic modulators and coupled into the transverse electric waveguide mode. The interference between the transmitted probe and the unshifted reference is detected at the corresponding radio frequency by two balanced photodiodes and a lock-in amplifier. This detection discriminates the probe and allows the measurement of its amplitude and phase. The delay time $\tau_{\mathrm{P}}$ between the prepump and pump pulse, and $\tau$ between pump and probe pulse (see Fig. 1) is controlled by optical path lengths using mechanical linear stages.

\section{RESULTS AND DISCUSSION}

The experiment is designed with the first pump pulse acting as strong prepump which fully depletes the GS gain via stimulated emission. In this way, the QDs interacting with the prepump are initialized to be close to transparency. The pulse energy of the prepump is chosen as the minimum value needed to deplete the GS gain, and is in the order of $\sim 0.5 \mathrm{pJ}$. After the delay time $\tau_{\mathrm{P}}$, the pump pulse of smaller energy $\sim 0.05 \mathrm{pJ}$ induces a gain depletion in the small- perturbation regime for the carrier distribution modified by the prepump. The resulting gain compression and recovery dynamics is monitored by the probe pulse of even smaller energy $\sim 0.01 \mathrm{pJ}$ at the delay time $\tau$ after the pump. The intensity of prepump and pump is modulated independently, hence we can sensitively detect the change in the gain $\delta g$ induced by the pump only. An example of how the prepump depletes the GS gain at $15 \mathrm{~K}$ is shown in the inset of Fig. 1, where $\delta g$ is measured as a function of the prepump pulse energy immediately after the prepump $\left(\tau_{\mathrm{P}}=\tau=0.2 \mathrm{ps}\right)$. We note that in the absorption regime at low-injection current (0.5 mA, $g_{0}=-10 \mathrm{~cm}^{-1}$, solid line in the inset of Fig. 1) the prepump drives the GS transition from absorption $(\delta g>0)$ to gain $(\delta g<0)$, revealing a coherent interaction regime. A similar crossover from gain to absorption is still observable in the gain regime $\left(10 \mathrm{~mA}, g_{0}=18 \mathrm{~cm}^{-1}\right.$, dashed line in the inset of Fig. 1), although of lower visibility, as expected from a reduction in the GS dephasing time in presence of injected carriers. ${ }^{16}$ The prepump energy used to drive the GS to transparency (see arrows in the inset) was chosen according to such measurements for each injection current and temperature.

Using a mean-field approach with rate equations for the dynamics of the carrier distribution functions, it was predicted $^{17}$ that the GS gain recovery in the presence of an optical pulse sequence is limited by the refill carrier dynamics in the ES and wetting layer since they act as a reservoir for the ultrafast GS recovery. ${ }^{7}$ As a result, after ultrafast amplification of the first pulse, the second pulse in the sequence generates a gain compression which recovers on a longer time scale if the delay between the two pulses is shorter than the recovery of the reservoir states. This effect would limit the maximum bit rate for optical pulse sequence amplification. Our experiments demonstrate that actually the opposite occurs. This is shown in Fig. 2 where the transient $\delta g$ (relative to the gain $g$ experienced by the pump in the presence of the prepump) versus $\tau$ is plotted for different delay times $\tau_{\mathrm{P}}$ at different injection currents and temperatures. ${ }^{18}$ We clearly observe a faster gain recovery after the prepump pulse at small $\tau_{\mathrm{P}}$, tending to the dynamics without prepump at large $\tau_{\mathrm{P}}$. This effect is especially pronounced at low temperatures when the redistribution of carriers among different dots via thermal escape into the wetting layer can be neglected. Remarkably though, even at room temperature at $10 \mathrm{~mA}$ injection current the gain recovery dynamics in the presence of prepump at the shortest $\tau_{\mathrm{P}}$ used in the experiment is still faster than without prepump.

In Refs. 14 and 16 we interpreted the gain recovery dynamics of QD-amplifiers at low temperature using a microstate model. In these works, single-pump probe differential transmission experiments for different injection current were fitted as a superposition of exponential responses having fixed decay times but varying amplitudes. This procedure models the macroscopic configuration as a superposition of microstates with given exponential dynamics but varying probability depending on the injection current. Using the same basic model, the dynamics observed in Fig. 2 can be understood in terms of a conditional recovery. Only microstates with a GS recovery faster than $\tau_{\mathrm{P}}$ will be sensitive to the second pump and contribute to $\delta g$ while the others are 


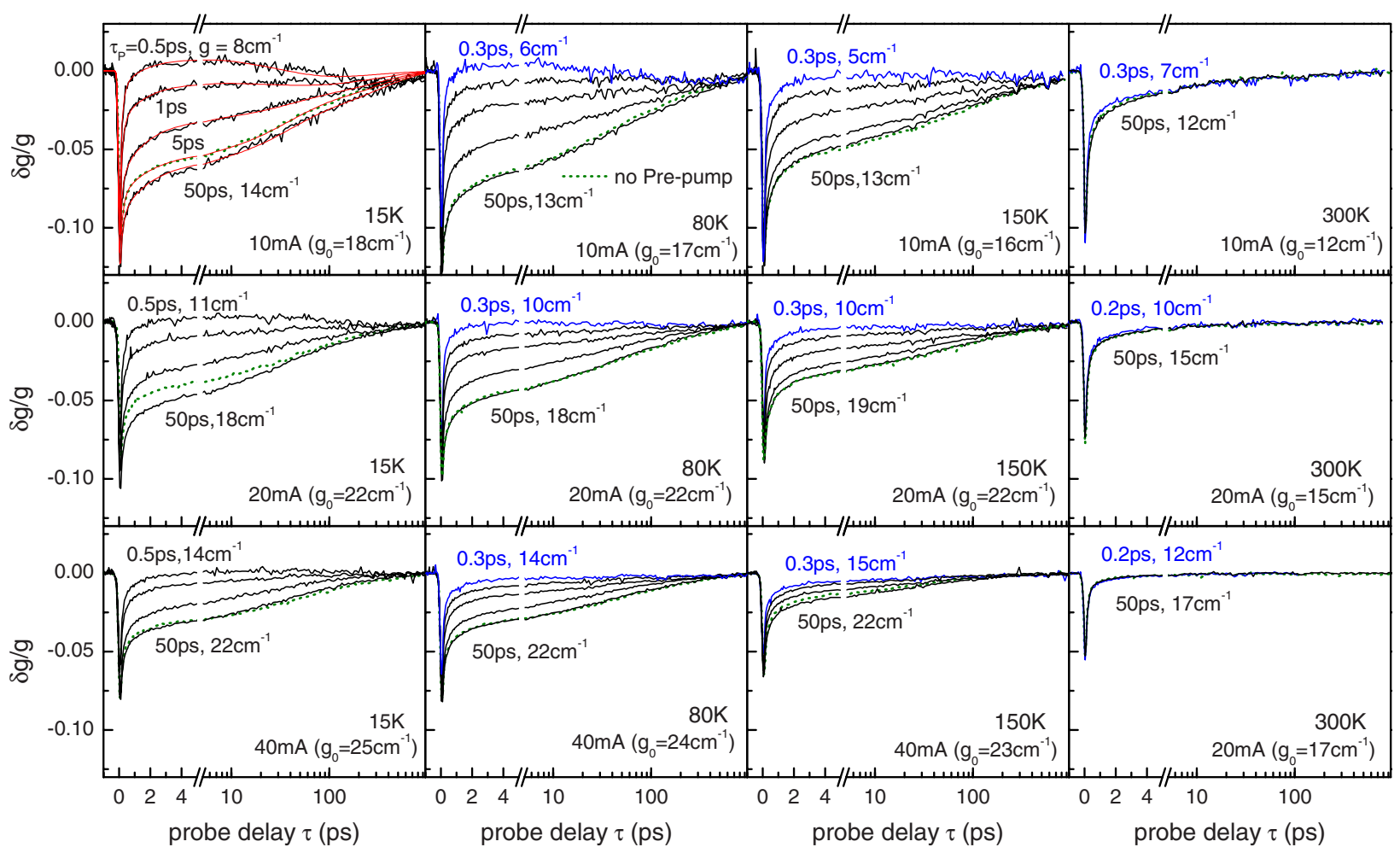

FIG. 2. (Color online) Differential change $\delta g$ of the gain induced by the second pump relative to the gain $g$ experienced by the second pump in the presence of the prepump for different delay times $\tau_{\mathrm{P}}$ between prepump and pump. The temperature, injection current, and modal gain without prepump $g_{0}$ for each set of measurements is indicated. Gain recovery dynamics in the absence of prepump are shown for comparison (dotted lines). On the top-left panel fit to the data are also shown (thin lines).

still transparent from the prepump depletion. Since the subensemble of microstates sensitive to the second pump is selected according to its fast GS recovery, the subsequent decay of $\delta g$ is faster than the gain recovery without prepump where all microstates are contributing. This concept is schematically shown in Fig. 3 where for simplicity microstates are grouped into three types, applicable to the situation at

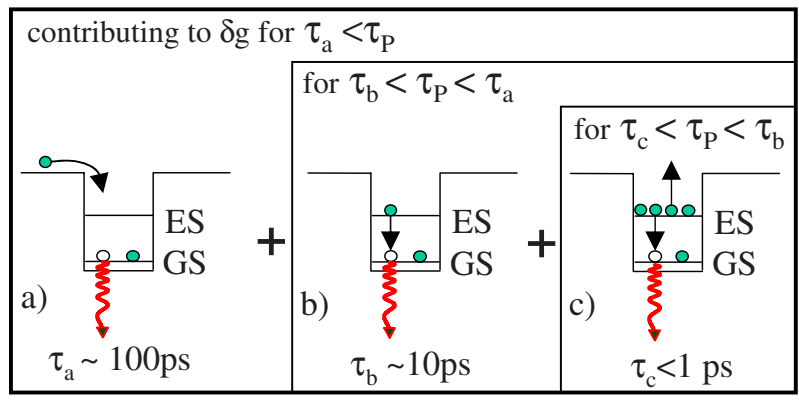

FIG. 3. (Color online) Sketch of the conditional gain recovery dynamics. Three types of microstates are depicted: (a) no carriers in the ES, (b) few carriers in the ES, and (c) many carriers in the ES. Microstates of type $\mathrm{b}$ and $\mathrm{c}$ have internal relaxation dynamics (black arrow) with time constants $\tau_{\mathrm{b}}$ and $\tau_{\mathrm{c}}$, respectively, to recover the GS depletion induced by the prepump (curly arrow) while microstates of type a recover on a longer time scale $\tau_{\text {a }}$ given by the interplay between carrier recombination and capture. For example, if $\tau_{\mathrm{c}}<\tau_{\mathrm{P}}<\tau_{\mathrm{b}}$, only microstates of type $\mathrm{c}$ have recovered the GS occupation and contribute to $\delta g$, i.e., the microstates a and b are suppressed as schematically shown by the black frames. low temperature where the carrier configuration in a QD is in its lowest energy state before the optical excitation: (a) no carriers in the ES, (b) few carriers in the ES, and (c) many carriers in the ES. After the prepump depletes the GS gain via stimulated emission (curly arrow in Fig. 3), the GS occupation recovers via intradot relaxation followed by recovery of the steady-state equilibrium given by the interplay between carrier recombination and injection. ${ }^{16}$ Microstates with no carriers in the ES show no intradot relaxation and contribute to the GS gain recovery only on a longer time scale, represented by $\tau_{\mathrm{a}} \sim 100$ ps. Microstates with few carriers in the ES show a faster GS gain recovery via phononmediated intradot dynamics represented by $\tau_{\mathrm{b}} \sim 10 \mathrm{ps}$. Microstates with many carriers in the ES have an ultrafast GS gain recovery due to the large number of intradot relaxation channels via Auger-type Coulomb interaction ${ }^{19}$ represented by $\tau_{\mathrm{c}}<1 \mathrm{ps}$. If $\tau_{\mathrm{c}}<\tau_{\mathrm{P}}<\tau_{\mathrm{b}}$ only microstates of type c contribute to $\delta g$, and its dynamics is governed by the intradot thermalization of this type of microstates, hence on the $\tau_{\mathrm{c}}$ time scale. With increasing $\tau_{\mathrm{P}}$ also microstates of type $\mathrm{b}$ (for $\tau_{\mathrm{P}}>\tau_{\mathrm{b}}$ ) and eventually type a (for $\tau_{\mathrm{P}}>\tau_{\mathrm{a}}$ ) contribute to $\delta g$, resulting in a slower recovery of $\delta g$ with increasing $\tau_{\mathrm{P}}$, as measured. One needs also to keep in mind that the recovery of $\delta g$ actually corresponds to the dynamics from microstates with one electron-hole pair less in the ES as a result of the prepump optical depletion. For microstates of type $\mathrm{c}$ this reduced ES reservoir has little influence on their intradot dynamics which remain ultrafast. However, when the other microstates come into play for $\tau_{\mathrm{P}}>\tau_{\mathrm{a}}$ one eventually does observe that the recovery of $\delta g$ is slower than in the absence 


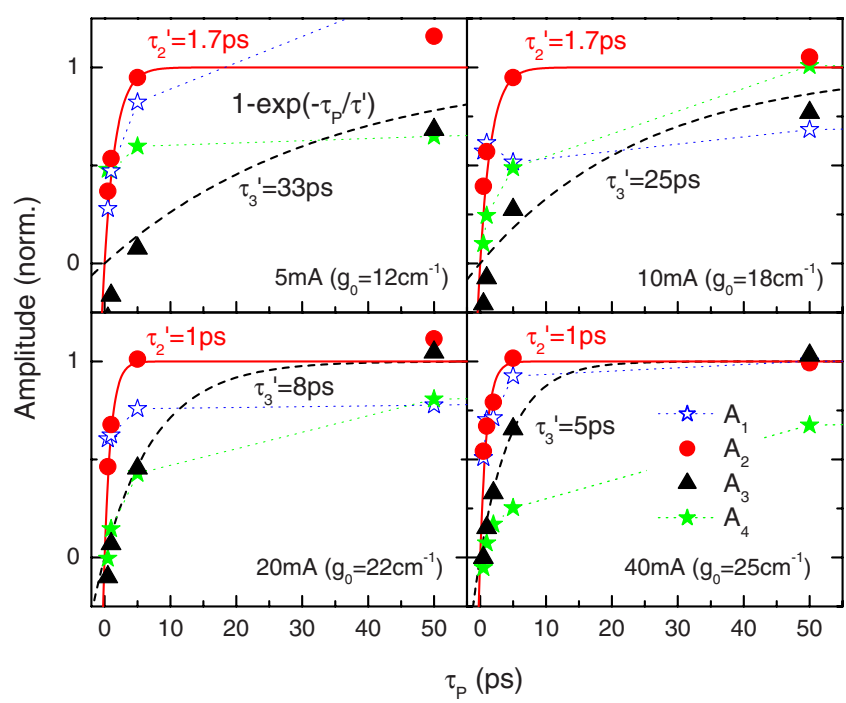

FIG. 4. (Color online) Amplitudes $A_{1,2,3,4}$ as function of $\tau_{\mathrm{P}}$ related to the time constants $\tau_{1,2,3,4}$ in the exponential fits to $\delta g$ at 15 $\mathrm{K}$ and various injection currents, as indicated. Amplitudes are normalized to their values without prepump, equivalent to $\tau_{\mathrm{P}} \rightarrow \infty$. The dependencies of $A_{2,3}$ are compared with $1-\exp \left(-\tau_{\mathrm{P}} / \tau_{2,3}^{\prime}\right)$ (solid and dashed lines) and the inferred values of $\tau_{2,3}^{\prime}$ are indicated. Dotted lines are connecting $A_{1,4}$.

of prepump. This is shown in Fig. 2 at $15 \mathrm{~K}$ when the level of injection current is sufficiently low to see a significant contribution from microstates with only few carriers.

Consistently with the microstate model discussed above, we have fitted $\delta g$ measured at $15 \mathrm{~K}$ for a series of $\tau_{\mathrm{P}}$ and injection currents using a four-component exponential response function of variable amplitudes $\left(A_{\mathrm{i}}, \mathrm{i}=1, \ldots, 4\right)$ but fixed time constants of $\tau_{1}=0.17 \mathrm{ps}, \tau_{2}=1.7 \mathrm{ps}, \tau_{3}=33 \mathrm{ps}$, and $\tau_{4}=330 \mathrm{ps}$ chosen to fit the whole series, which determines them to $\sim 10 \%$ uncertainty. Examples of fits to the data are shown in Fig. 2 top left. The dependence of the amplitudes on $\tau_{\mathrm{P}}$ is shown in Fig. 4. Remarkably, the amplitudes of $\tau_{2}$ (circles) and $\tau_{3}$ (triangles) are scaling with 1 $-\exp \left(-\tau_{\mathrm{P}} / \tau_{2,3}^{\prime}\right)$, which is the probability that a microstate with exponential dynamics of decay time $\tau_{2,3}^{\prime}$ has recovered after the delay $\tau_{\mathrm{P}}$ following the initial GS depletion by the prepump. In other words, they scale as expected from a conditional recovery after the prepump. For the amplitudes of $\tau_{1}$ and $\tau_{4}$, we do not find such a dependence. For the $\tau_{4}$ time scale this is expected since it is given not by the intradot dynamics but by the recovery of the equilibrium carrier density via recombination and injection, hence it is common to all QDs and independent of the specific microstate. $\tau_{1}$ instead is close to our temporal resolution, and can be influenced by instantaneous contributions such as two-photon absorption. ${ }^{16,20}$ We note that $\tau_{2,3}^{\prime}$ is somewhat smaller than $\tau_{2,3}$, for example, for $g_{0}=25 \mathrm{~cm}^{-1}$ (Fig. 4 bottom right) $\tau_{2}^{\prime}=1$ ps and $\tau_{3}^{\prime}=5$ ps. Also we find that $\tau_{2,3}^{\prime}$ depends on the injection current. This observation can be understood considering two effects. First, microstates contributing to $\delta g$ after the recovery from the prepump GS depletion will have one electron-hole pair less in the ES and hence their internal dynamics measured by the gain recovery after the second pump represented by $\tau_{2,3}$ will be slightly slower than $\tau_{2,3}^{\prime}$ due to the reduced ES reservoir. Second, even at low temperature a large number of microstates contribute to the macroscopic response, particularly at high injection currents. The fourfold exponential fit does not capture each individual microstate but represents only a coarse division into microstate types. The inferred $\tau^{\prime}$ hence represents a mixture of contributions from the superposition of subensembles of microstates which changes with injection current.

\section{CONCLUSIONS}

In conclusion, using a dual-pump probe technique we provide direct evidence that the gain recovery dynamics in electrically pumped InGaAs/GaAs quantum dots is a superposition of intradot relaxation dynamics from microstates with different discrete number of carriers, rather than the dynamics of an average carrier number commonly employed in mean-field theory. The effect manifests as a gain recovery in the presence of a strong optical prepump which is faster than without prepump. This phenomenon, opposite to the expectations from the commonly used rate equation models with mean-field carrier distribution functions, is the result of a conditional gain recovery where microstates with fast internal dynamics are selected by probing shortly after the prepump. The effect is particularly evident at low temperature but is still present at room temperature, beneficial for practical applications aiming at the ultrafast amplification of an optical pulse sequence for high-speed optical signal processing.

\section{ACKNOWLEDGMENTS}

This work was supported by the UK Engineering and Physical Sciences Research Council (EPSRC) under Grant No. EP/E056385. The sample was grown by Innolume GmbH (Dortmund, Germany) and processed by M. Rossetti and A. Fiore (EPFL Lausanne, Switzerland).

\footnotetext{
*borrip@cf.ac.uk

${ }^{1}$ Nano-Optoelectronics: Concepts, Physics and Devices, edited by M. Grundmann (Springer, New York, 2002).

${ }^{2}$ H. C. Schneider, W. W. Chow, and S. W. Koch, Phys. Rev. B 64, 115315 (2001).

${ }^{3}$ W. W. Chow and S. W. Koch, IEEE J. Quantum Electron. 41,
}

495 (2005)

${ }^{4}$ J. Gomis-Bresco, S. Dommers, V. Temnov, U. Woggon, M. Laemmlin, D. Bimberg, E. Malic, M. Richter, E. Schöll, and A. Knorr, Phys. Rev. Lett. 101, 256803 (2008).

${ }^{5}$ M. Grundmann and D. Bimberg, Phys. Rev. B 55, 9740 (1997).

${ }^{6}$ I. O'Driscoll, P. Blood, and P. Smowton, IEEE J. Quantum Elec- 
tron. 46, 525 (2010).

${ }^{7}$ P. Borri, W. Langbein, S. Schneider, U. Woggon, R. L. Sellin, D. Ouyang, and D. Bimberg, IEEE J. Sel. Top. Quantum Electron. 8, 984 (2002).

${ }^{8}$ M. Abbarchi, C. Mastrandrea, T. Kuroda, T. Mano, A. Vinattieri, K. Sakoda, and M. Gurioli, J. Appl. Phys. 106, 053504 (2009).

${ }^{9}$ R. Osovsky, D. Cheskis, V. Kloper, A. Sashchiuk, M. Kroner, and E. Lifshitz, Phys. Rev. Lett. 102, 197401 (2009).

${ }^{10}$ E. Dekel, D. Regelman, D. Gershoni, E. Ehrenfreund, W. V. Schoenfeld, and P. M. Petroff, Phys. Rev. B 62, 11038 (2000).

${ }^{11}$ T. Kuroda, S. Sanguinetti, M. Gurioli, K. Watanabe, F. Minami, and N. Koguchi, Phys. Rev. B, 66, 121302(R) (2002).

${ }^{12}$ C. Santori, G. S. Solomon, M. Pelton, and Y. Yamamoto, Phys. Rev. B 65, 073310 (2002).

${ }^{13}$ V. Cesari, W. Langbein, P. Borri, M. Rossetti, A. Fiore, S. Mikhrin, I. Krestnikov, and A. Kovsh, Appl. Phys. Lett. 90, 201103 (2007).
${ }^{14}$ V. Cesari, W. Langbein, and P. Borri, Appl. Phys. Lett. 94, 041110 (2009).

${ }^{15}$ P. Borri, S. Schneider, W. Langbein, and D. Bimberg, J. Opt. A: Pure Appl. Opt. 8, S33 (2006).

${ }^{16}$ P. Borri, W. Langbein, S. Schneider, U. Woggon, R. L. Sellin, D. Ouyang, and D. Bimberg, Phys. Rev. Lett. 89, 187401 (2002).

${ }^{17}$ T. W. Berg, S. Bischoff, I. Magnusdottir, and J. Mørk, IEEE Photon. Technol. Lett. 13, 541 (2001).

${ }^{18}$ Measurements of $\delta g$ for different $\tau_{\mathrm{P}}$ are taken using the same input pump energy and are normalized to the average pump intensity propagating through the waveguide, as detailed in Ref. 20.

${ }^{19}$ T. R. Nielsen, P. Gartner, and F. Jahnke, Phys. Rev. B 69, 235314 (2004).

${ }^{20}$ V. Cesari, W. Langbein, P. Borri, M. Rossetti, A. Fiore, S. Mikhrin, I. Krestnikov, and A. Kovsh, IET Optoelectron. 1, 298 (2007). 